\title{
Teaching Multivariable Control Using the Quadruple-Tank Process ${ }^{1} 2$
}

\author{
Karl Henrik Johansson ${ }^{\dagger}$, Alexander Horch ${ }^{\ddagger}$, Olle Wijk ${ }^{\ddagger}$, and Anders Hansson ${ }^{\ddagger}$ \\ $\dagger$ Department of Electrical Engineering and Computer Sciences \\ University of California, Berkeley, CA 94720-1770, U.S.A. \\ johans@eecs . berkeley.edu \\ †Department of Signals, Sensors and Systems \\ Royal Institute of Technology, S-100 44 Stockholm, Sweden \\ $\{$ horchlollel hansson dee.kth.se
}

\begin{abstract}
A recent multivariable laboratory process is presented together with its use in a graduate control course. The process is called the Quadruple-Tank Process and demonstrates a multivariable level control problem. The multivariable zero dynamics of the system can be made both minimum phase and nonminimum phase by simply changing a valve. This makes the QuadrupleTank Process suitable for illustrating many concepts in linear and nonlinear multivariable control. In this paper some of these are described together with the basic setup of the process. Two computer exercises and one laboratory exercise have been developed as part of a course in multivariable and nonlinear control. These are detailed and some experience from the course is presented.
\end{abstract}

\section{Introduction}

Multivariable control is today taught in most control curricula. This includes both linear and nonlinear design methods. Recently it has been an increased interest in emphasizing the limitations the process imposes on control designs $[5,3,19]$. This is natural since new technologies have opened possibilities for integrated solutions, where old control configurations may be changed to achieve an enhanced overall performance. A good control engineering course should be accompanied by hands-on experiments [2, 13, 1]. It is, however, not trivial to find pedagogical examples to illustrate multivariable performance limitations in feedback systems.

\footnotetext{
${ }^{1}$ The work by Karl Henrik Johansson was supported by the Swedish Foundation for International Cooperation in Research and Higher Education.

${ }^{2}$ IEEE Conference on Decision and Control, Phoenix, AZ, 1999.
}

There exists only a handful of multivariable laboratory processes, available from manufactures such as Quanser Consulting, Educational Control Products, Feedback Instruments, and TecQuipment. None of these illustrate the effect of multivariable zeros on the closed-loop control performance.

In this paper we describe a laboratory process, which was designed to illustrate the importance of multivariable zeros and how the zeros may change due to variations in the process. The process is called the Quadruple-Tank Process $[8,11,10]$ and is a level control problem based on four interconnected water tanks and two pumps. The inputs to the process are the voltages to the two pumps and the outputs are the water levels in the lower two tanks. The Quadruple-Tank Process can easily be built by using standard equipment available in many control laboratories. The setup is thus simple, but still the process can illustrate several interesting multivariable phenomena. One of its main features is that the zero dynamics can be made minimum phase or nonminimum phase by simply changing a valve. For the linearized model of the process, both the zero location and the zero direction have direct physical interpretations. Also the relative gain array has a straightforward meaning for the process. The Quadruple-Tank Process is thus suitable to use in teaching fundamentals of multivariable control.

The Quadruple-Tank Process was developed and built 1996 at Lund Institute of Technology, Sweden, in order to illustrate the importance of multivariable zero location for control design [8]. Since then it has been used extensively in the control curriculum in Lund. A number of student projects as part of courses in adaptive control, system identification, and real-time control have been performed on the Quadruple-Tank Process. These projects consist of modeling from physical or experimental data, control design, simulation, 
implementation, and evaluation together with a short written and oral presentation. The Quadruple-Tank Process has also been the topic for master thesis projects. Nunes [17] demonstrated decentralized PID control. Automatic tuning of multivariable PID controllers based on relay feedback was studied by Recica [18]. As a CalTech summer project, Grebeck [7] investigated what performance that was achievable for the Quadruple-Tank Process using various optimal control methods. He concluded that in the minimumphase setting for the process there was no advantage of using centralized multivariable control, while in the nonminimum-phase setting $H_{\infty}$ control gave better responses. The Quadruple-Tank Process is also used in the education at the Royal Institute of Technology in Stockholm, as described in the paper, and at University of Delaware. Other research institutes are currently copying the design and there is also ongoing commercial development.

The outline of the paper is as follows. A nonlinear model for the Quadruple-Tank Process based on physical data is derived in Section 2. The location and the direction of a multivariable zero of the linearized model are derived in Section 3. It is shown that the valve positions of the process uniquely determine if the system is minimum phase or nonminimum phase. The relative gain array is discussed in Section 4. Section 5 gives an overview of our experiences in using the QuadrupleTank Process in teaching. Some concluding remarks are given in Section 6. More details on the Quadruple-Tank Process are found in [10], see also [8, 11, 9].

\section{The Quadruple-Tank Process}

In this section we derive a physical model for the Quadruple-Tank Process. A schematic diagram of the process is shown in Figure 1. The target is to control the level in the lower two tanks with two pumps. The process inputs are $v_{1}$ and $v_{2}$ (input voltages to the pumps) and the outputs are $y_{1}$ and $y_{2}$ (voltages from level measurement devices). Mass balances and Bernoulli's law yield

$$
\begin{aligned}
& \frac{d h_{1}}{d t}=-\frac{a_{1}}{A_{1}} \sqrt{2 g h_{1}}+\frac{a_{3}}{A_{1}} \sqrt{2 g h_{3}}+\frac{\gamma_{1} k_{1}}{A_{1}} v_{1}, \\
& \frac{d h_{2}}{d t}=-\frac{a_{2}}{A_{2}} \sqrt{2 g h_{2}}+\frac{a_{4}}{A_{2}} \sqrt{2 g h_{4}}+\frac{\gamma_{2} k_{2}}{A_{2}} v_{2}, \\
& \frac{d h_{3}}{d t}=-\frac{a_{3}}{A_{3}} \sqrt{2 g h_{3}}+\frac{\left(1-\gamma_{2}\right) k_{2}}{A_{3}} v_{2}, \\
& \frac{d h_{4}}{d t}=-\frac{a_{4}}{A_{4}} \sqrt{2 g h_{4}}+\frac{\left(1-\gamma_{1}\right) k_{1}}{A_{4}} v_{1},
\end{aligned}
$$

where $A_{i}$ is the cross-section area of Tank $i, a_{i}$ the cross-section area of the outlet hole, and $h_{i}$ the water level. The voltage applied to Pump $i$ is $v_{i}$ and the corresponding flow is $k_{i} v_{i}$. The parameters $\gamma_{1}, \gamma_{2} \in[0,1]$

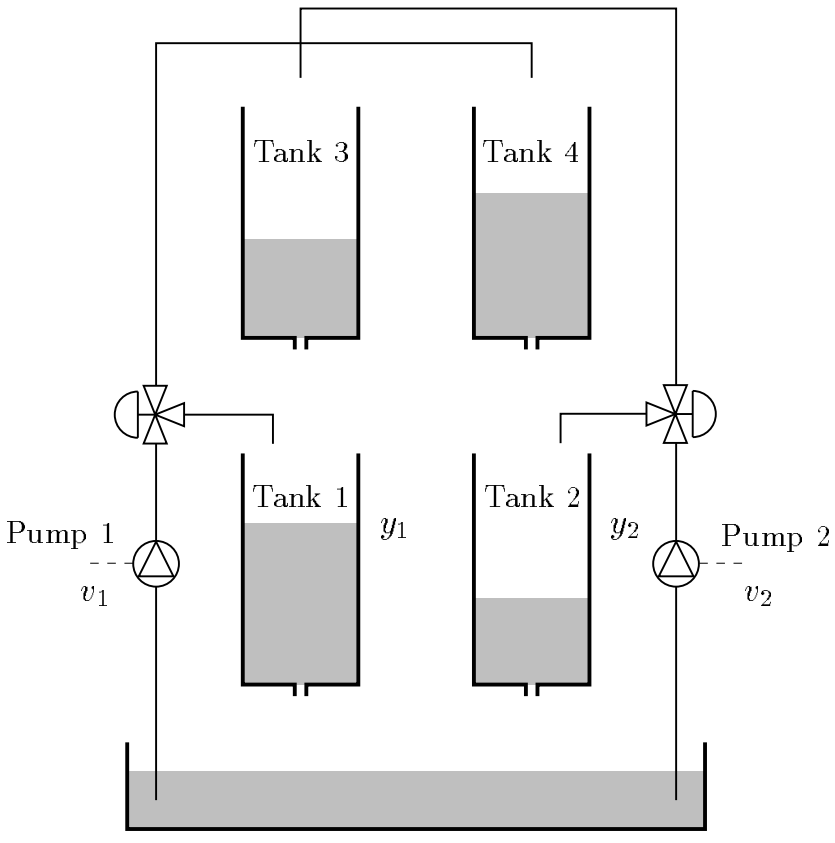

Figure 1: Schematic diagram of the Quadruple-Tank Process. The water levels in Tank 1 and Tank 2 are controlled by two pumps. The positions of the valves determine the location of a multivariable zero for the linearized model. The zero can be put in either the left or the right half-plane.

are determined from how the valves are set prior to an experiment. The flow to Tank 1 is $\gamma_{1} k_{1} v_{1}$ and the flow to Tank 4 is $\left(1-\gamma_{1}\right) k_{1} v_{1}$ and similarly for Tank 2 and Tank 3 . The acceleration of gravity is denoted $g$. The measured level signals are $y_{1}=k_{c} h_{1}$ and $y_{2}=k_{c} h_{2}$.

The transfer matrix of the linearized system is

$$
G(s)=\left[\begin{array}{cc}
\frac{\gamma_{1} c_{1}}{1+s T_{1}} & \frac{\left(1-\gamma_{2}\right) c_{1}}{\left(1+s T_{3}\right)\left(1+s T_{1}\right)} \\
\frac{\left(1-\gamma_{1}\right) c_{2}}{\left(1+s T_{4}\right)\left(1+s T_{2}\right)} & \frac{\gamma_{2} c_{2}}{1+s T_{2}}
\end{array}\right]
$$

where the time constants are

$$
T_{i}=\frac{A_{i}}{a_{i}} \sqrt{\frac{2 h_{i}^{0}}{g}}, \quad i=1, \ldots, 4
$$

$c_{1}=T_{1} k_{1} k_{c} / A_{1}$, and $c_{2}=T_{2} k_{2} k_{c} / A_{2}$. Note the way the parameters $\gamma_{1}$ and $\gamma_{2}$ enter the transfer matrix. Particularly, we see that if either $\gamma_{1}=1$ or $\gamma_{2}=1$, the transfer matrix is triangular and has no finite zeros. This corresponds to that the flow through one of the valves are directed only to the corresponding lower tank. Physical data for the process are given in [10]. 


\section{Physical Interpretation of Zero}

The zero locations and their directions of the transfer matrix (1) are considered in this section. It is shown that they have intuitive physical interpretations in terms of how the valves $\gamma_{1}$ and $\gamma_{2}$ are set.

\section{Zero location}

The zeros of $G$ are the zeros of the numerator polynomial of the rational function

$$
\begin{aligned}
\operatorname{det} G(s) & =\frac{c_{1} c_{2}}{\gamma_{1} \gamma_{2} \prod_{i=1}^{4}\left(1+s T_{i}\right)} \\
& \times\left[\left(1+s T_{3}\right)\left(1+s T_{4}\right)-\frac{\left(1-\gamma_{1}\right)\left(1-\gamma_{2}\right)}{\gamma_{1} \gamma_{2}}\right]
\end{aligned}
$$

It follows that the system is nonminimum phase for

$$
0<\gamma_{1}+\gamma_{2}<1
$$

and minimum phase for

$$
1<\gamma_{1}+\gamma_{2}<2
$$

The multivariable zero being in the left or in right halfplane has a straightforward physical interpretation. Let $q_{i}$ denote the flow through Pump $i$ and assume that $q_{1}=q_{2}$. Then the sum of the flows to the upper tanks is $\left[2-\left(\gamma_{1}+\gamma_{2}\right)\right] q_{1}$ and the sum of the flows to the lower tanks is $\left(\gamma_{1}+\gamma_{2}\right) q_{1}$. Hence, the flow to the lower tanks is greater than the flow to the upper tanks if the system is minimum phase. The flow to the lower tanks is smaller than the flow to the upper tanks if the system is nonminimum phase. It is intuitively easier to control $y_{1}$ with $v_{1}$ and $y_{2}$ with $v_{2}$, if most of the flows goes directly to the lower tanks. There is thus an immediate connection between zero location and physical intuition. The control problem is particularly hard if the total flow going to the left tanks (Tanks 1 and 3 ) is approximately equal to the total flow going to the right tanks (Tanks 2 and 4 ). This corresponds to $\gamma_{1}+\gamma_{2} \approx 1$, i.e., a multivariable zero close to the origin.

\section{Zero direction}

An important difference between scalar systems and multivariable systems is that not only the location of a multivariable zero is important but also its direction. We define the (output) direction of a zero $z$ as a vector $\psi \in \mathbf{R}^{2}$ of unit length such as $\psi^{T} G(z)=0$. If $\psi$ is equal to a unit vector, then the zero is only associated with one output. If this is not the case, then the effect of a right half-plane zero may be distributed between both outputs. In that sense, a multivariable right half-plane zero must not deteriorate the performance as much as a corresponding scalar zero. For the transfer matrix $G$ in (1), the zero direction of a zero $z>0$ is given by $\psi=\left(\psi_{1}, \psi_{2}\right)^{T}$ such that

$$
\psi^{T}\left[\begin{array}{cc}
\frac{\gamma_{1} c_{1}}{1+z T_{1}} & \frac{\left(1-\gamma_{2}\right) c_{1}}{\left(1+z T_{3}\right)\left(1+z T_{1}\right)} \\
\frac{\left(1-\gamma_{1}\right) c_{2}}{\left(1+z T_{4}\right)\left(1+z T_{2}\right)} & \frac{\gamma_{2} c_{2}}{1+z T_{2}}
\end{array}\right]=0 .
$$

Note that it follows from this equation that $\psi_{1}, \psi_{2} \neq 0$, so the zero is never associated with only one output. If we solve the equation for $\gamma_{2}$ and simplify, it follows that

$$
\frac{\psi_{1}}{\psi_{2}}=-\frac{1-\gamma_{1}}{\gamma_{1}} \cdot \frac{c_{2}\left(1+z T_{1}\right)}{c_{1}\left(1+z T_{4}\right)\left(1+z T_{2}\right)} .
$$

From this equation it is possible to conclude that if $\gamma_{1}$ is small, then $z$ is mostly associated with the first output. If $\gamma_{1}$ is close to one, then $z$ is mostly associated with the second output. Hence, for a given zero location, the relative size of $\gamma_{1}$ and $\gamma_{2}$ determines which output the right half-plane zero is related to.

The relation between $\gamma_{1}$ and $\gamma_{2}$ and the zero location $z$ and the zero direction $\left(\psi_{1}, \psi_{2}\right)^{T}$ can be described as a map $\left(\gamma_{1}, \gamma_{2}\right) \mapsto\left(z, \psi_{1} / \psi_{2}\right)$. Each valve position $\left(\gamma_{1}, \gamma_{2}\right)$ defines a unique zero configuration $\left(z, \psi_{1} / \psi_{2}\right)$. In this way, any zero location and direction can be tested, or equivalently one valve knob can be used to choose zero location and the other zero direction.

\section{Relative Gain Array}

The relative gain array (RGA) was introduced by Bristol [4] as a measure of interaction in multivariable control systems. The RGA $\Lambda$ is defined as $\Lambda=G(0) *$ $G^{-T}(0)$, where the asterisk denotes the Schur product (element-by-element matrix multiplication) and $-T$ inverse transpose. It is possible to show that the elements of each row and column of $\Lambda$ sum up to one, so for a $2 \times 2$ system the RGA is determined by the scalar $\lambda=\Lambda_{11}$. The RGA is used as a tool mainly in the process industry to decide on control structure issues such as inputoutput pairing for decentralized controllers [16, 12, 20]. McAvoy [14] proposed that one should strive for a pairing with $0.67<\lambda<1.50$. The system is particularly hard to control if $\lambda<0$.

The RGA of the Quadruple-Tank Process is easily derived to be given by

$$
\lambda=\frac{\gamma_{1} \gamma_{2}}{\gamma_{1}+\gamma_{2}-1} .
$$

Note that the RGA is only depending on the valve settings. Figure 2 shows a contour plot of $\lambda$ as a function of $\gamma_{1}$ and $\gamma_{2}$. The edges of the box $\gamma_{1}, \gamma_{2} \in[0,1]$ corresponds to $\lambda=0$ and $\lambda=1$, respectively, as is shown in the figure. The magnitude of $\lambda$ increases as $\gamma_{1}+\gamma_{2}$ 


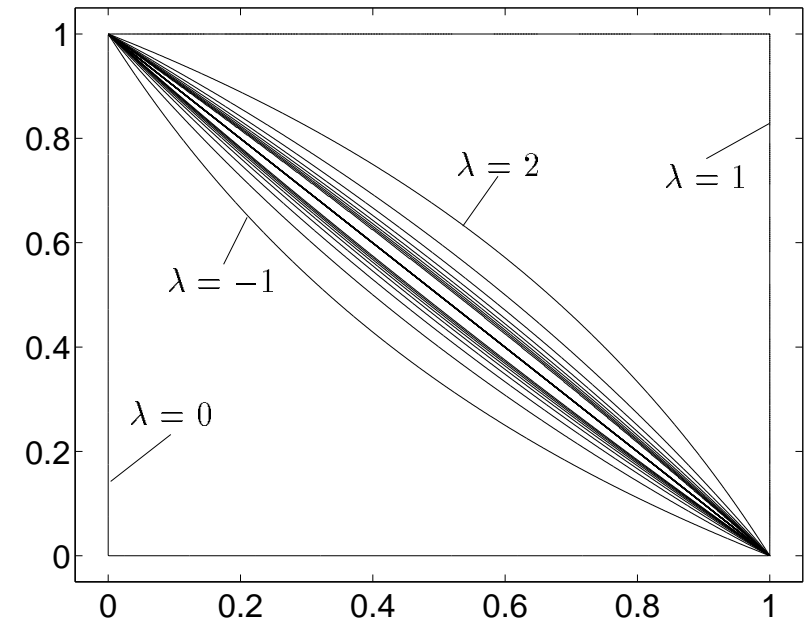

Figure 2: Contour plot of the RGA $\lambda$ as a function of the valve parameters $\gamma_{1}$ and $\gamma_{2}$. The plot is drawn for $\lambda=-10,-9, \ldots, 10$. We have $\lambda \leq 0$ below the straight line between $(0,1)$ and $(1,0)$ and $\lambda \geq 1$ above the line. Close to the line, $|\lambda|$ is large.

becomes close to one. There are no $\gamma_{1}, \gamma_{2} \in(0,1)$ so that $\lambda \in(0,1)$. From (2) we see for instance that $\lambda<0$ if $\gamma_{1}+\gamma_{2}<1$, which corresponds to the nonminimumphase setting discussed previously.

If the valves of the quadruple-tank process are set such that $\gamma_{1}+\gamma_{2}<1$, then the RGA analysis suggests that the input-output pairing $v_{1}-y_{2}$ and $v_{2}-y_{1}$ should be chosen for decentralized control. Let

$$
\widetilde{G}=\left[\begin{array}{ll}
G_{21} & G_{22} \\
G_{11} & G_{12}
\end{array}\right]
$$

be the linearized model with $y_{1}$ and $y_{2}$ permuted. The RGA for $\widetilde{G}$ is

$$
\tilde{\lambda}=\frac{\left(1-\gamma_{1}\right)\left(1-\gamma_{2}\right)}{1-\gamma_{1}-\gamma_{2}}
$$

Hence, if $\gamma_{1}+\gamma_{2}<1$ then $\tilde{\lambda}>0$ so a decentralized control structure corresponding to $\widetilde{G}$ is preferable according to the RGA. This is intuitive from physical considerations, because $\gamma_{1}+\gamma_{2}<1$ means that more water is flowing to the upper tanks than directly to the lower ones. Decentralized control is then easier using $v_{1}$ to control $y_{2}$ and $v_{2}$ to control $y_{1}$, than vice versa.

\section{Teaching Experiences}

Recently a control course in multivariable and nonlinear control was developed at the Royal Institute of Technology, where the Quadruple-Tank Process is used extensively throughout the course. The prerequisites is a basic course in control. The recent textbook [6], which covers both linear and nonlinear control techniques, is used. Two computer exercises and a laboratory exercise based on the Quadruple-Tank Process are part of the course. They are described next.

\section{Computer Exercise 1}

In the first computer exercise the process dynamics is investigated by computing the poles and zeros of the system. The singular value frequency response is investigated as well as the RGA. Step responses are conducted in order to verify the coupling predicted by the RGA. Comparisons are made between the minimum-phase case and the nonminimum-phase case. The conclusion is that the coupling is severe for the nonminimum-phase case. Then decentralized PI control is investigated. A design is made by pairing the inputs and outputs of the process as suggested by the RGA. The PI controller is tuned such that for the minimumphase case the cross-over frequency is $0.1 \mathrm{rad} / \mathrm{sec}$ with a phase margin of $80^{\circ}$ and for the nonminimum-phase case $0.006 \mathrm{rad} / \mathrm{sec}$ with $60^{\circ}$. The singular values of the sensitivity and complementary sensitivity functions are plotted in order to verify performance and robustness. The designs are further evaluated in simulations and the coupling between the loops are investigated. It is seen that the interaction is large for the nonminimumphase case.

\section{Computer Exercise 2}

In the second computer exercise PI controller designs based on decoupling are investigated followed by the method of Glover-McFarlane [15] to robustify a nominal design. Both static and dynamic decoupling are investigated. It is noticed that dynamic decoupling is superior for the minimum-phase case. The reason for this is that the static decoupling is poor for frequencies close to the desired cross-over frequency of the loop transfer function. For the nonminimum-phase case static decoupling is superior, because the static decoupling is good enough for the lower bandwidth. At the end of the second computer exercise Glover-McFarlane's method is used to robustify the design. Plotting the singular values of the sensitivity and complementary sensitivity functions verifies the improvement, see Figure 3. The figure shows the sensitivity and the complementary sensitivity functions for both the minimum-phase and the nonminimum-phase setting. Statically decoupled PI control as well as the robustified Glover-McFarlane design are presented. Only the maximum singular values are shown. The minimum-phase setting is almost a magnitude faster than the nonminimum-phase setting. (Note the different frequency scales.) This illustrates the limitation on closed-loop bandwidth that a multivariable right half-plane zero imposes. 

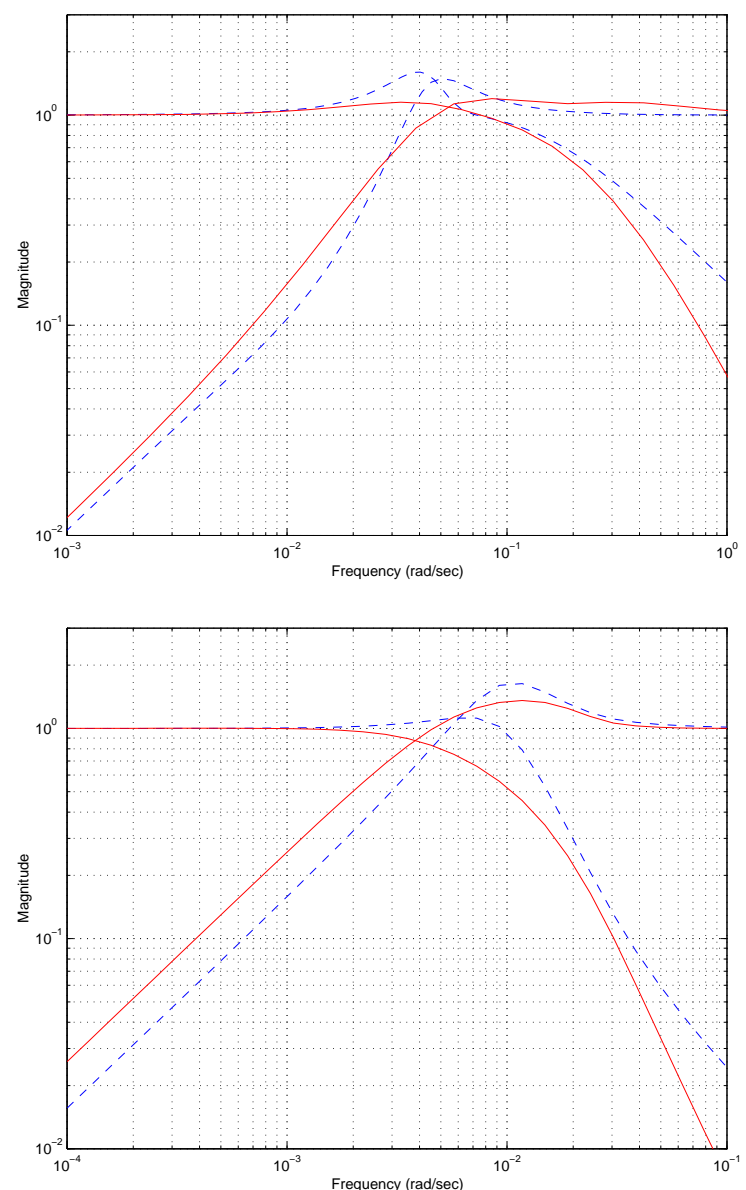

Figure 3: Sensitivity functions and complementary sensitivity functions for the minimum-phase setting (upper diagram) and the nonminimum-phase setting (lower diagram). PI control designs with static decoupling (dashed) and GloverMcFarlane designs (solid) are shown. Note that the bandwidth for the nonminimum-phase case is almost a magnitude lower than for the minimum-phase case. This is due to the performance limitation that the multivariable right half-plane zero imposes.

\section{Laboratory Exercise}

In the laboratory exercise the students are asked to verify the theoretical results of this paper. Then experiments are performed in order to determine the physical parameters of the process, such as the valve settings and the proportional constants relating the pump voltages to the water flows. Then the designs from the computer exercises are redone using the identified physical model of the process. Manual control is investigated as well as decentralized and statically decoupled PI control. Both the minimum-phase and the nonminimumphase cases are considered. As an example, Figure 4 shows experiments for both these cases when statically decoupled PI control is applied. Note how much slower the responses for the nonminimum-phase process are
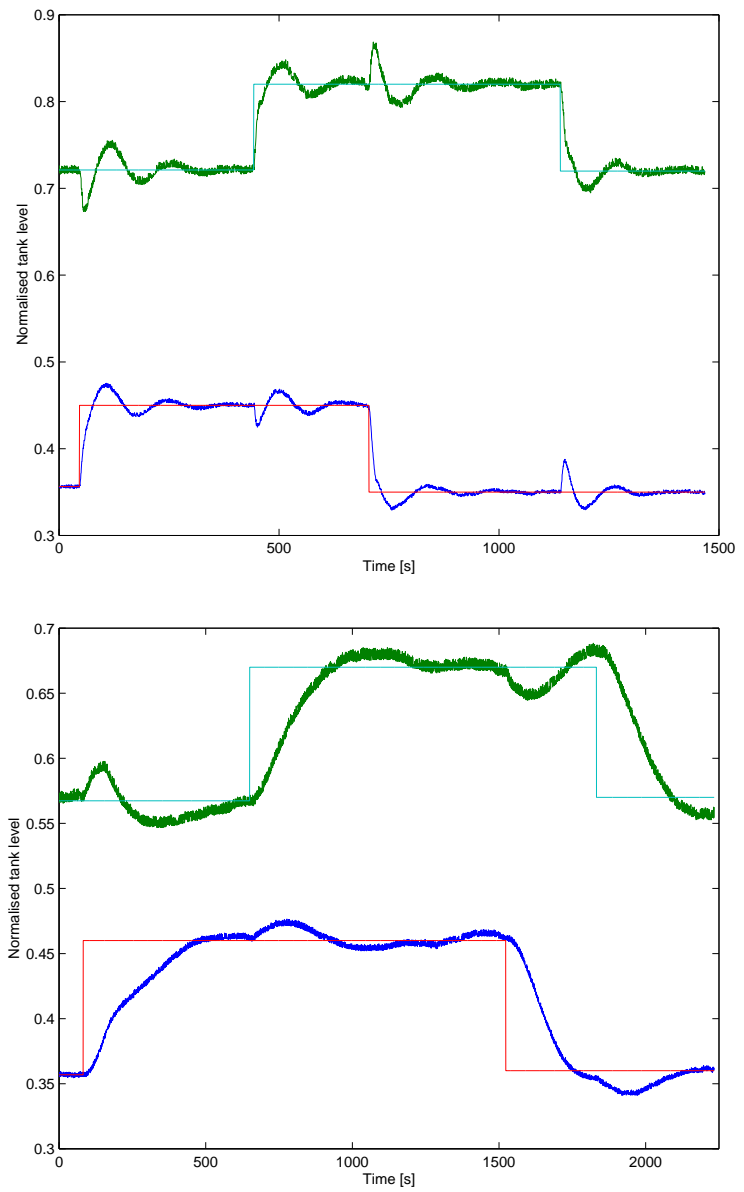

Figure 4: Experiments for the minimum-phase (upper diagram) and nonminimum-phase (lower diagram) process controlled by statically decoupled PI controllers. The levels $y_{2}$ of Tank 2 (upper curves) and $y_{1}$ of Tank 1 (lower curves) are shown together with their setpoints. Tank levels equal to one correspond to full tanks.

as compared to the minimum-phase process.

The experiments illustrate for the students that multivariable control is important if process limitations such as right half-plane zeros are present. They also show that if there are no tight limitations, then scalar PI control in each loop is sufficient. Added to these, the exercise clarify that there might be fundamental constraints due to the process design that no linear feedback controller can remove.

\section{Conclusions}

The Quadruple-Tank Process has been presented. It is a recent laboratory process that was designed in order to illustrate various concepts in multivariable control. In particular, it is suited to demonstrate performance limitations in multivariable control design caused by 
right half-plane zeros. This follows from that the process has a multivariable zero that in a direct way is connected to the physical positions of two valves. The positions are given by the parameters $\gamma_{1}, \gamma_{2} \in(0,1)$. It was shown in the paper that $\gamma_{1}+\gamma_{2}$ determines the location of the zero, so that if $\gamma_{1}+\gamma_{2}<1$ the system is nonminimum phase and if $\gamma_{1}+\gamma_{2}>1$ the system is minimum phase. The quotient $\gamma_{1} / \gamma_{2}$ gives the zero direction.

Some examples of how the Quadruple-Tank Process has been used in teaching have also been presented. The conclusion of the teaching experiences is that the Quadruple-Tank Process is very well suited for demonstrating both the effects of coupling and performance limitations in multivariable control systems. A particular course on multivariable control was described. Computer exercises were shown to be a good preparation for a laboratory exercise. A remark on the laboratory experiments is that since the closed loop bandwidth for the nonminimum-phase case is low, the experiments take a relatively long time to conduct. It is therefore crucial to implement the controllers such that the transfer between manual and automatic mode is bumpless. We also found it important to inform the students about the time the different response experiments take. Prior to that some of the students thought that something was wrong with their design and thus interrupted the experiments to start to trouble shooting, although they were only experiencing the large difference between setpoint response times for minimum-phase and nonminimum-phase processes.

\section{References}

[1] K. J. Åström and M. Lundh. Lund control program combines theory with hands-on experience. IEEE Control Systems Magazine, 12(3):22-30, 1992.

[2] K. J. Åström and A.-B. Östberg. A teaching laboratory for process control. IEEE Control Systems Magazine, 6:37-42, October 1986.

[3] S. P. Boyd and C. H. Barratt. Linear Controller Design-Limits of Performance. Prentice-Hall, Englewood Cliffs, NJ, 1991.

[4] E. Bristol. On a new measure of interaction for multivariable process control. IEEE Transactions on Automatic Control, 11:133, 1966.

[5] J. Freudenberg and D. Looze. Frequency Domain Properties of Scalar and Multivariable Feedback Systems. Springer-Verlag, Berlin, Germany, 1988.

[6] T. Glad and L. Ljung. Control Theory: Multivariable and Nonlinear Methods. Studentlitteratur, Lund, 1997. In Swedish.

[7] M. Grebeck. A comparison of controllers for the quadruple tank system. Technical report, Department of Automatic Control, Lund Institute of Technology, Sweden, 1998.

[8] K. H. Johansson. Relay feedback and multivariable control. PhD thesis, Department of Automatic Control, Lund Institute of Technology, Sweden, November 1997.

[9] K. H. Johansson. Interaction bounds in multivariable control systems. In IFAC World Congress, Beijing, China, 1999.

[10] K. H. Johansson. The Quadruple-Tank Process-A multivariable laboratory process with an adjustable zero. IEEE Control Systems Technology, 2000. To appear.

[11] K. H. Johansson and J. L. R. Nunes. A multivariable laboratory process with an adjustable zero. In 17 th American Control Conference, Philadelphia, PA, 1998.

[12] J. M. Maciejowski. Multivariable Feedback Design. Addison-Wesley, Reading, MA, 1989.

[13] M. Mansour and W. Schaufelberger. Software and laboratory experiment using computers in control education. IEEE Control Systems Magazine, 9(3):1924, 1989 .

[14] T. J. McAvoy. Interaction Analysis: Principles and Applications. Instrument Society of America, Research Triangle Park, NC, 1983.

[15] D. McFarlane and K. Glover. A loop shaping design procedure using $H_{\infty}$ synthesis. IEEE Transactions on Automatic Control, 37(6):759-769, 1992.

[16] M. Morari and E. Zafiriou. Robust Process Control. Prentice-Hall, Englewood Cliffs, NJ, 1989.

[17] J. L. R. Nunes. Modeling and control of the Quadruple-Tank Process. Master thesis, Department of Automatic Control, Lund Institute of Technology, Sweden, 1997.

[18] V. Recica. Automatic tuning of multivariable controllers. Master thesis, Department of Automatic Control, Lund Institute of Technology, Sweden, 1998.

[19] M. M. Seron, J. H. Braslavsky, and G. C. Goodwin. Fundamental Limitations in Filtering and Control. Springer-Verlag, 1997.

[20] S. Skogestad and I. Postlethwaite. Multivariable Feedback Control-Analysis and Design. John Wiley \& Sons, 1996. 